\title{
Lentivirus-mediated PHLDA2 overexpression inhibits trophoblast proliferation, migration and invasion, and induces apoptosis
}

\author{
FENG JIN, CHONG QIAO, NANNAN LUAN and HUI LI \\ Department of Obstetrics and Gynecology, Shengjing Hospital \\ of China Medical University, Shenyang, Liaoning 110004, P.R. China
}

Received July 30, 2015; Accepted January 7, 2016

DOI: $10.3892 /$ ijmm.2016.2508

\begin{abstract}
Inadequate trophoblast invasion and increased trophoblast apoptosis cause serious pregnancy complications. Pleckstrin homology-like domain, family A, member 2 (PHLDA2) has been linked to fetal size at birth and growth restriction in a number of studies. However, the impact of PHLDA2 on trophoblast function had not been studied previously, to the best of our knowledge. In the present study, immunofluorescence staining demonstrated that primary trophoblasts isolated from placental villous tissues were positive for cytokeratin 18 (CK18), vimentin and human placental lactogen (hPL). JEG-3 cells and primary trophoblasts were infected with lentivirus overexpressing PHLDA2. RT-qPCR and western blot analysis detected high levels of PHLDA2. A Cell Counting Kit-8 (CCK-8) assay showed that PHLDA2 overexpression inhibited trophoblast proliferation. In addition, PHLDA2 significantly induced apoptosis, as evidenced by Annexin V-FITC/propidium iodide (PI) and Hoechst staining, along with activation of Bax and caspase-3 and also decreased Bcl-2 expression. Further investigation showed that PHLDA2 effectively induced reactive oxygen species (ROS) generation, caused cytochrome $c$ release from the mitochondria into the cytosol and decreased mitochondrial membrane potential. PHLDA2 likely induced apoptosis through the mitochondrial pathway. Wound healing and Transwell assays indicated that PHLDA2 overexpression efficiently suppressed cell migration and invasion. These data suggest that PHLDA2 plays an important role in the occurrence and development of pregnancy complications by promoting trophoblast apoptosis and suppressing cell invasion.
\end{abstract}

Correspondence to: Dr Hui Li, Department of Obstetrics and Gynecology, Shengjing Hospital of China Medical University, 36 Sanhao Street, Shenyang, Liaoning 110004, P.R. China E-mail: huililh246@163.com

Key words: pleckstrin homology-like domain, family A, member 2 overexpression, trophoblasts, JEG-3, lentivirus, invasion, apoptosis

\section{Introduction}

Pregnancy is both a complex and unique physiological process that involves many events, including successful implantation, decidualization, placentation and parturition (1). The placenta is an organ whcih grows in the uterus and connects the fetus to the uterine wall of the mother; it allows not only for the uptake of nutrients, but also for the exchange of gases and the transport of metabolic waste $(2,3)$. Invasion of extravillous trophoblasts into the uterine decidua and myometrium occurs within the first 20 weeks of gestation. This is of great importance to maternal circulation at the maternal-fetal interface during pregnancy $(4,5)$. Inadequate trophoblast invasion into the uterine spiral arteries and impaired placentation affect fetal growth and lead to serious pregnancy complications, such as intrauterine growth restriction (IUGR), preeclampsia (PE), preterm birth and spontaneous abortion (6,7). Additionally, excessive trophoblast apoptosis can also be observed in the placentas of patients who have been affected by pregnancy complications $(5,8)$. However, the precise mechanisms remain unclear.

Pleckstrin homology-like domain, family A, member 2 (PHLDA2; also known as BRW1C, BWR1C, HLDA2, IPL and TSSC3), a maternally expressed imprinted gene (9), is negatively correlated with size at birth and has been linked to fetal growth restriction in a number of studies (10-13). In the placentae of babies affected by IUGR $(10,14,15)$ and with low birth weight (LBW) (12), upregulated expression of PHLDA2 has been observed. In a mouse model, it was noted that knockdown of PHLDA2 resulted in a significant increase in placental size during gestation (16). Previous research has also shown that downregulated PHLDA2 expression in onecell zygotes using siRNA resulted in accelerated blastocyst development (17). Therefore, high expression of PHLDA2 may have a close correlation with abnormal placental function and pregnancy complications. However, the effects of PHLDA2 on trophoblast invasion and cell apoptosis, which play a critical role in the onset and development of pregnancy complications, have yet to be fully clarified.

In the present study, we isolated primary trophoblasts and detected cytokeratin 18 (CK18), vimentin and human placental lactogen (hPL) expression. The human placental choriocarcinoma cell line JEG-3 and primary trophoblasts were infected with lentiviruses, and subsequently we examined the impact 
of PHLDA2 overexpression on cell proliferation, apoptosis, mitochondrial function, migration and invasion.

\section{Materials and methods}

Tissue collection. Human villous tissues were obtained from women who underwent artificial abortion within 7 weeks of pregnancy. Written informed consent was signed by the patients who were recruited to the study. Ethics approval was provided by The China Medical University Ethics Committee (Shenyang, China).

Primary culture of primary trophoblasts and cell culture. The human placental villous tissues were first rinsed in PBS and minced into $1-2 \mathrm{~mm}^{3}$ fragments, placed in a $35-\mathrm{mm}$ culture dish and subsequently subjected to sequential 10-min treatments with $0.125 \%$ trypsin at $37^{\circ} \mathrm{C}$. The supernatant was collected, and then Dulbecco's Modified Eagle's medium (DMEM) (Gibco, Grand Island, NY, USA) containing fetal bovine serum (FBS) (HyClone, Logan, UT, USA) was added in order to terminate the digestion. The cell suspension was filtered through a $70-\mu \mathrm{m}$ mesh nylon strainer to remove undigested tissue fragments. The filtrate was centrifuged, washed with PBS and cultured in minimum essential medium (MEM) for $48 \mathrm{~h}$. The adherent cells were primary trophoblasts.

Human placental choriocarcinoma JEG-3 cells were purchased from the Chinese Academy of Sciences Cell Bank (Shanghai, China). The cells were cultured in DMEM supplemented with 10\% FBS and incubated in a humidified atmosphere with $5 \% \mathrm{CO}_{2}$ at $37^{\circ} \mathrm{C}$.

Treatment groups. Primary trophoblasts and JEG-3 cells that were transfected with lentivirus containing control vector or lentivirus overexpressing PHLDA2 were termed Vector or PHLDA2. Primary trophoblasts and JEG-3 cells, which were not transfected with lentiviruses, were used as controls in our experiments.

Immunofluorescence staining. In the present study, the primary trophoblast slides were first fixed with $4 \%$ paraformaldehyde for $15 \mathrm{~min}$ and then permeabilized with $0.1 \%$ Triton X-100 for $30 \mathrm{~min}$ at room temperature. Goat serum (purchased from Solarbio, Beijing, China) was added in order to block non-specific binding sites, followed by incubation with CK18 antibody (dilution 1:50; \#sc-6259; Santa Cruz Biotechnology, Inc., Santa Cruz, CA, USA), vimentin antibody (dilution 1:100; \#WL0274; Wanleibio, Shenyang, China) and hPL antibody (dilution 1:100; \#ab11396; Abcam, Cambridge, MA, USA) at $4^{\circ} \mathrm{C}$ overnight. The slides were then washed three times with PBS for 5 min and incubated with Cy3-labeled goat anti-rabbit secondary antibody (\#A0516) or Cy3-labeled goat anti-mouse secondary antibody (\#A0521) (Beyotime Institute of Biotechnology, Haimen, China) for $1 \mathrm{~h}$ at room temperature. Subsequently, 4',6-diamidino-2-phenylindole (DAPI) was added in order to stain nuclei, and they were then examined by fluorescence microscopy (BX53; purchased from Olympus, Tokyo, Japan).

Generation of overexpressing cell lines. Lentivirus overexpressing PHLDA2 or containing control vector was purchased from HanBio (Shanghai, China). JEG-3 cells and primary trophoblasts were cultured in 6-well plates. After reaching $70 \%$ confluence, medium containing lentiviruses and polybrene $(8 \mu \mathrm{g} / \mathrm{ml}$; Hanbio) was added at a multiplicity of infection (MOI) of 10 and mixed with the cells. Polybrene was used to improve infection efficiency. After incubation for $24 \mathrm{~h}$, supernatants in the wells were replaced by DMEM containing FBS and cultured for 24 and $48 \mathrm{~h}$ for subsequent analyses.

Reverse transcription-quantitative PCR (RT-qPCR). Total RNA was isolated from the cells in each group with an RNAsimple Total RNA kit (Tiangen Biotech Co., Ltd., Beijing, China) and reverse transcription was carried out. RT-qPCR was performed using SYBR-Green Master Mix (Solarbio) on an Exicycler ${ }^{\mathrm{TM}} 96$ quantitative fluorescence analyzer (Bioneer Corporation, Daejeon, Korea). The reaction conditions were as follows: $95^{\circ} \mathrm{C}$ for $10 \mathrm{~min} ; 40$ cycles of $95^{\circ} \mathrm{C}$ for $10 \mathrm{sec}, 60^{\circ} \mathrm{C}$ for $20 \mathrm{sec}$ and $72^{\circ} \mathrm{C}$ for $30 \mathrm{sec}$. Gene expression levels were calculated using the $2^{-\Delta \Delta \mathrm{Ct}}$ method, as previously described (18). $\beta$-actin was used as the normalization internal control and sequences of primers are shown in Table I.

Cell Counting Kit-8 (CCK-8) cell proliferation assay. Cell proliferation was detected by CCK-8 (Beyotime Institute of Biotechnology) according to the manufacturer's instructions. In brief, cells were seeded in 96-well plates at a density of $2 \times 10^{3}$ cells/well. CCK- 8 reagent was added at $0,24,48$ and $72 \mathrm{~h}$. The plates were incubated for an additional $1 \mathrm{~h}$ at $37^{\circ} \mathrm{C}$. The absorbance at $450 \mathrm{~nm}$ was measured with a microplate reader (ELX-800; BioTek, Winooski, VT, USA).

Western blot analysis. After the cells were lysed using NP-40 lysis buffer (Beyotime Institute of Biotechnology), total proteins were extracted from the supernatants of cell lysates. In addition, cells were collected and ice-cold PBS was used to resuspend the pelleted cell. Mitochondrial and cytosolic proteins were isolated with a Cell Mitochondria Isolation kit (Beyotime Institute of Biotechnology). Subsequently, $1 \mathrm{ml}$ mitochondrial lysis buffer containing phenylmethanesulfonyl fluoride (PMSF) was added to incubate with the isolated mitochondria, and the mitochondrial proteins were obtained. Protein concentrations were determined using a BCA protein assay kit (Beyotime Institute of Biotechnology). Equal amounts of proteins $(40 \mu \mathrm{g})$ were separated on sodium dodecyl sulfate-polyacrylamide gel electrophoresis (SDS-PAGE) and then transferred to PVDF membranes (Millipore Corp., Bedford, MA, USA). The membranes were incubated overnight at $4^{\circ} \mathrm{C}$ with PHLDA2 antibody (dilution 1:400; \#bs-6884R; BIOSS, Beijing, China), cleaved-caspase-3 antibody (dilution 1:1,000; \#WL0146), Bax antibody (dilution 1:1,000; \#WL0101), Bcl-2 antibody (dilution 1:1,000; \#WL0104) and cytochrome $c$ antibody (dilution 1:1,000; \#WL0483; Wanleibio). We also used COX IV antibody (\#WL0933; Wanleibio) and $\beta$-actin (\#WL0001; Wanleibio). COX IV was used as a loading control for mitochondrial proteins and $\beta$-actin for cytosolic proteins. After being washed with TTBS buffer, the membranes were incubated with HRP-conjugated goat anti-rabbit IgG secondary antibody (dilution 1:5,000; \#A0208; Beyotime Institute of Biotechnology) for $45 \mathrm{~min}$ at $37^{\circ} \mathrm{C}$. Band intensities were determined using Gel-Pro Analyzer software. 
Table I. Genes and primer sequences.

\begin{tabular}{llcr}
\hline Gene & \multicolumn{1}{c}{ Primer sequence } & Accession no. & Temp $\left({ }^{\circ} \mathrm{C}\right)$ \\
\hline PHLDA2 & F: CCATCCTCAAGGTGGACTGC & NM_003311.3 & 59.9 \\
\multirow{2}{*}{-actin } & R: TTCCTGGCGGCTGCGAAAGT & & 67.5 \\
& F: CCATCGTCCACCGCAAAT & NM_001101.3 & 59.5 \\
& R: GCTGTCACCTTCACCGTTC & & 55.6 \\
\hline
\end{tabular}

F, forward; R, reverse; PHLDA2, pleckstrin homology-like domain, family A, member 2.

Analysis of apoptosis by flow cytometry. Cells were trypsinized, counted and seeded. Subsequently, cells were harvested and stained with Annexin V-FITC/propidium iodide (PI) (KeyGen, Nanjing, China) according to the manufacturer's instructions. Briefly, cells were washed twice with PBS and resuspended in binding buffer. Cells were subsequently incubated with $5 \mu \mathrm{l}$ Annexin V-FITC and $5 \mu \mathrm{l}$ PI for $15 \mathrm{~min}$ at room temperature in the dark and analyzed by flow cytometry (BD Accuri C6; Becton-Dickinson, Franklin Lakes, NJ, USA).

Hoechst staining. After $24 \mathrm{~h}$ infection, cells from each group were cultured on the slides in 12-well plates at a density of $1 \times 10^{5}$ cells/slide. When cells reached $80 \%$ confluence (cultured for approximately $24 \mathrm{~h}$ ), the supernatant was discarded and they were washed twice with PBS. Cells were then fixed for $20 \mathrm{~min}$ at room temperature. Hoechst solution (Beyotime Institute of Biotechnology) was added, and cells were observed and photographed under a microscope (AE31; Motic, Xiamen, China).

Measurement of reactive oxygen species (ROS) using 2'7'-dichlorofluorescin diacetate (DCFH-DA). Intracellular ROS levels were measured using a Reactive Oxygen Species Assay kit (Beyotime Institute of Biotechnology) and flow cytometry (C6; Becton-Dickinson). In brief, cells in each group were plated in a T25 culture flask at a density of $5 \times 10^{5}$ cells/flask. When they reached $70 \%$ confluence, the supernatant was discarded and $2 \mathrm{ml}$ of diluted DCFH-DA (10 $\mu \mathrm{M}$ final concentration) solution was added to each well and then incubated at $37^{\circ} \mathrm{C}$ for $20 \mathrm{~min}$ and washed with serum-free medium to remove extracellular DCFH-DA. The cells were harvested, washed, resuspended in PBS and then detected by flow cytometry.

Mitochondrial membrane potential assay. Mitochondrial membrane potential (also known as $\Delta \Psi \mathrm{m}$ ) was studied using MitoTracker Red CMXRos (Invitrogen Life Technologies, Grand Island, NY, USA) according to the manufacturer's instructions. In brief, cells were harvested and labeled with MitoTracker (a dilution of $1: 200$ ) at $37^{\circ} \mathrm{C}$ for $20 \mathrm{~min}$. Cells were harvested, washed twice with PBS and resuspended in PBS. Mitochondrial membrane potential was analyzed by flow cytometry (FACSCalibur; Becton-Dickinson).

Wound healing assay. Cells in each group were cultured in 6-well plates. Subsequently, the supernatant was aspired and a wound was created with a sterile $200-\mu 1$ pipette tip. The cells were then washed twice with serum-free medium (Gibco) to remove cell debris and photographed immediately. After $24 \mathrm{~h}$ of incubation, photographic images were acquired under an inverted microscope (AE31; Motic) and the migration distance was calculated. The cell migration rates were calculated using the following formula: cell migration rate $(\%)=(1-$ the distance following healing/the distance prior to healing) x 100\%. Each experiment was repeated three times.

Transwell assay. A Transwell chamber (Corning Life Sciences, Tewksbury, MA, USA) pre-coated with Matrigel gel (Becton-Dickinson) was placed into a 24-well plate. After incubation for $2 \mathrm{~h}$ at $37^{\circ} \mathrm{C}$, the Matrigel gel solidified. Cells in each group were digested with trypsin, resuspended in serum-free medium, and then added to the upper chamber ( $2 \times 10^{4}$ cells/well). Subsequently, $800 \mu 1$ medium containing $20 \%$ FBS was added to the lower chamber. After incubation for $24 \mathrm{~h}$ at $37^{\circ} \mathrm{C}$, the cells which had not invaded were removed with a cotton swab. The invading cells in the lower chamber were fixed with paraformaldehyde at room temperature for $20 \mathrm{~min}$ and stained with hematoxylin staining solution for $5 \mathrm{~min}$. Five fields were randomly selected and the number of invading cells was counted under an inverted microscope (AE31; Motic).

Statistical analysis. All experiments were performed three times. Data are presented as the means \pm SD $(n=3$ replicates for each analysis). All analyses were performed using GraphPad Prism 5.0 software (GraphPad Software, Inc., San Diego, CA, USA). One-way ANOVA followed by the Bonferroni post hoc test was performed. A P-value $<0.05$ was considered to indicate a statistically significant difference.

\section{Results}

Isolation and characterization of primary trophoblasts. We examined the expression of CK18, vimentin and hPL in the isolated cells. Immunofluorescence staining demonstrated that CK18 (Fig. 1A), vimentin (Fig. 1B) and hPL (Fig. 1C) were highly expressed, suggesting that we successfully isolated and characterized primary trophoblasts.

Establishment of cell lines overexpressing PHLDA2. JEG-3 cells and primary trophoblasts were infected with lentivirus. RT-qPCR and western blot analysis were used to identify the increased expression of PHLDA2 in lentivirus-infected JEG-3 cells and lentivirus-infected primary trophoblasts at 48-h post-infection. As shown in Fig. 2A and B, PHLDA2 mRNA and protein expression levels in the JEG-3 cells infected with PHLDA2-overexpressing lentivirus were 3.94-fold $(\mathrm{P}<0.01)$ 
A
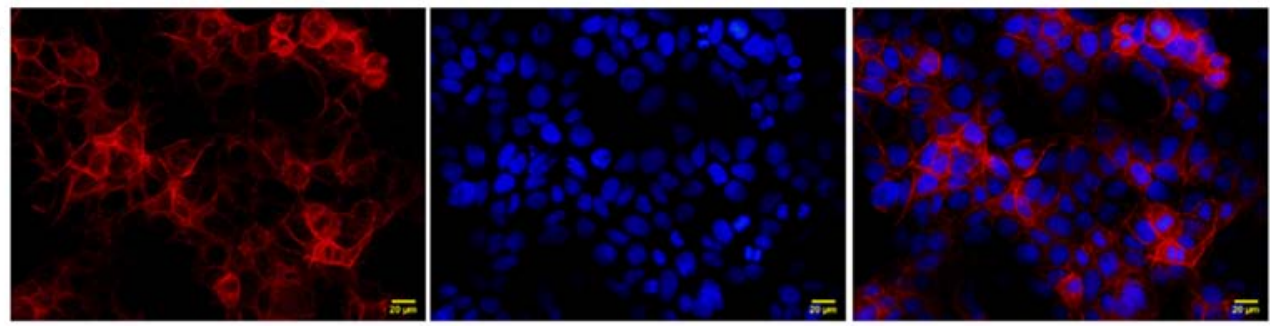

CK18

DAPI

Merged

B
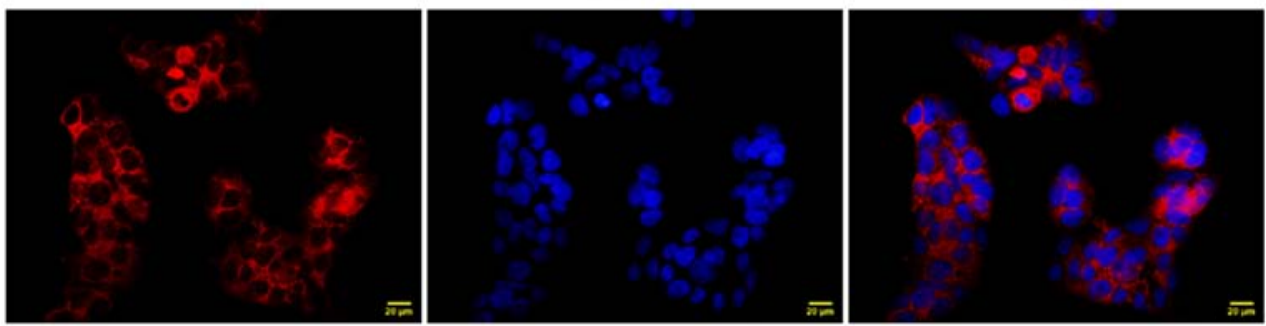

Vimentin

DAPI

Merged

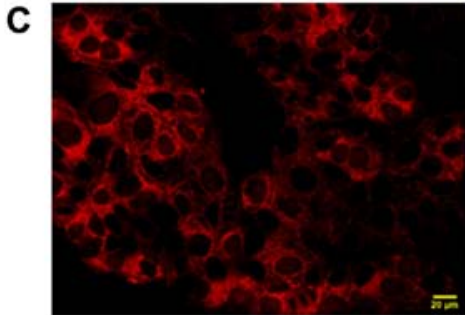

$\mathrm{hPL}$

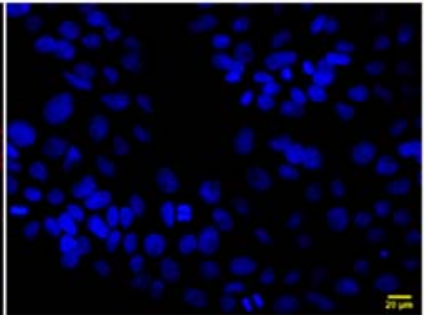

DAPI

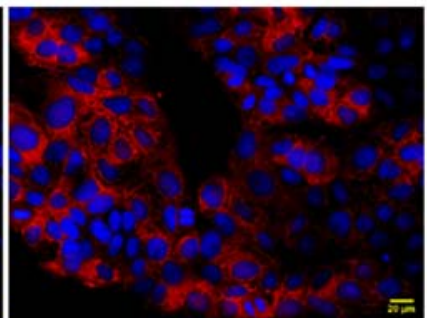

Merged

Figure 1. Expression of cytokeratin 18 (CK18), vimentin and human placental lactogen (hPL) in primary trophoblasts. Cell slides were fixed, permeabilized, and incubated with primary antibodies and Cy3-labelled secondary antibodies. CK18 were stained red by immunofluorescence staining. The nuclei were stained blue. (A) CK18, (B) vimentin, and (C) hPL expression. Representative images are shown. Scale bar, $20 \mu \mathrm{m}$.

A

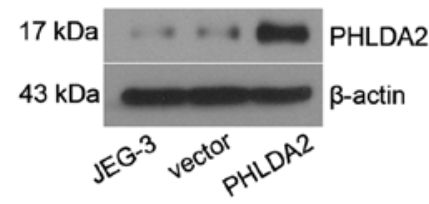

B

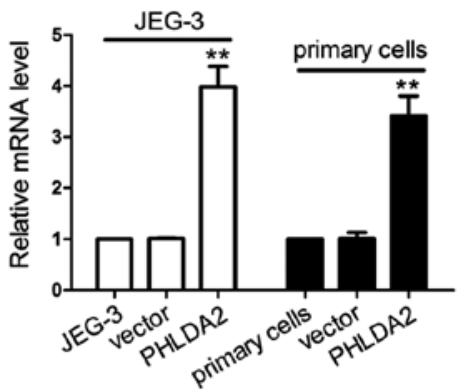

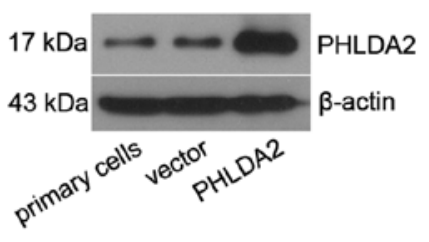

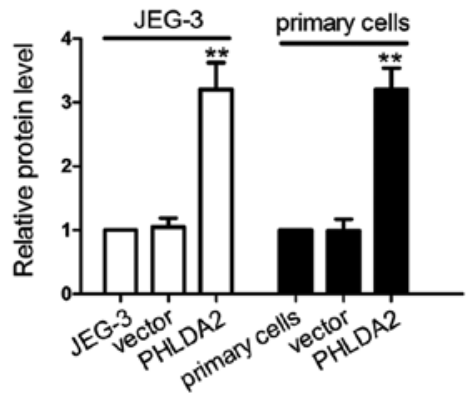

C

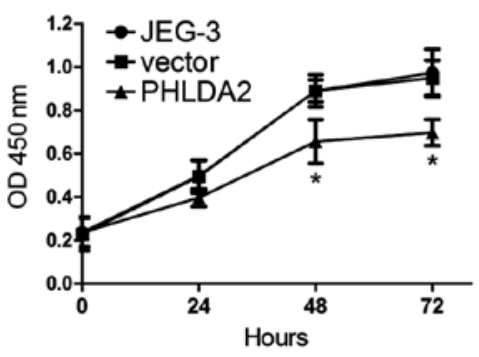

Figure 2. Establishment of pleckstrin homology-like domain, family A, member 2 (PHLDA2) overexpressing cell lines; PHLDA2 overexpression inhibits cell proliferation. (A) JEG-3 cells and primary trophoblasts were infected with lentivirus overexpressing PHLDA2 or containing control vector. Total proteins were extracted after $48 \mathrm{~h}$ of infection and the concentrations were measured. Subsequently, western blot analysis was performed to detect the expression of PHLDA2 in JEG-3 cells and primary trophoblasts. PHLDA2 protein expression was normalized to $\beta$-actin expression. (B) Total RNA was isolated from the cells in each group after $48 \mathrm{~h}$ of infection. RT-qPCR was performed to detect PHLDA2 mRNA expression in JEG-3 cells and primary trophoblasts. (C) JEG-3 cells and primary trophoblasts were seeded in a 96-well plate. The effects of PHLDA2 on cell proliferation ability were analyzed by CCK-8 assay at $0,24,48$ and $72 \mathrm{~h}$. The results are expressed as the means $\pm \mathrm{SD}$. ${ }^{*} \mathrm{P}<0.05$ and ${ }^{* *} \mathrm{P}<0.01$, compared with the control vector groups. 
A
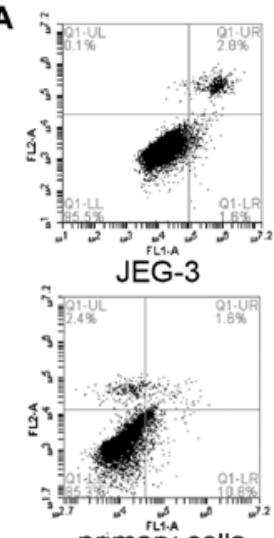

primary cells

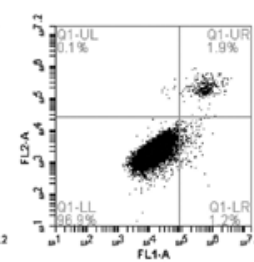

vector

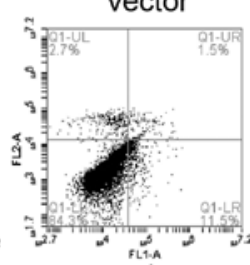

vector

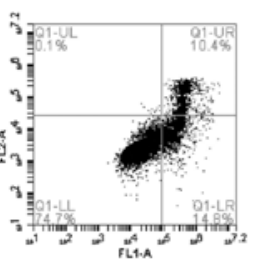

PHLLA'D2

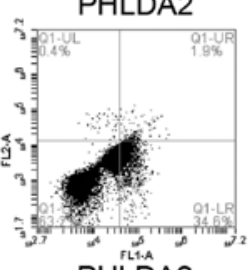

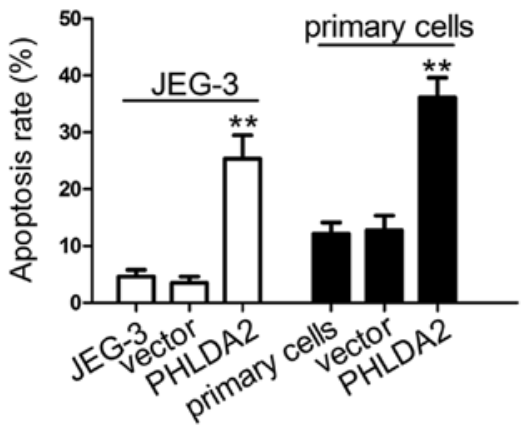

PHLDA2

B
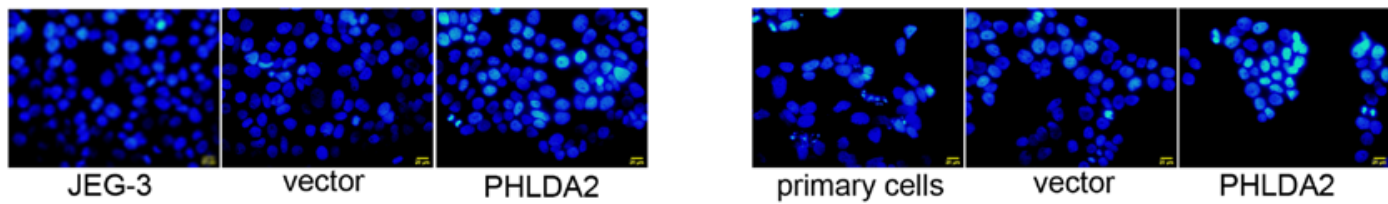

primary cells $\quad$ vector $\quad$ PHLDA2

C
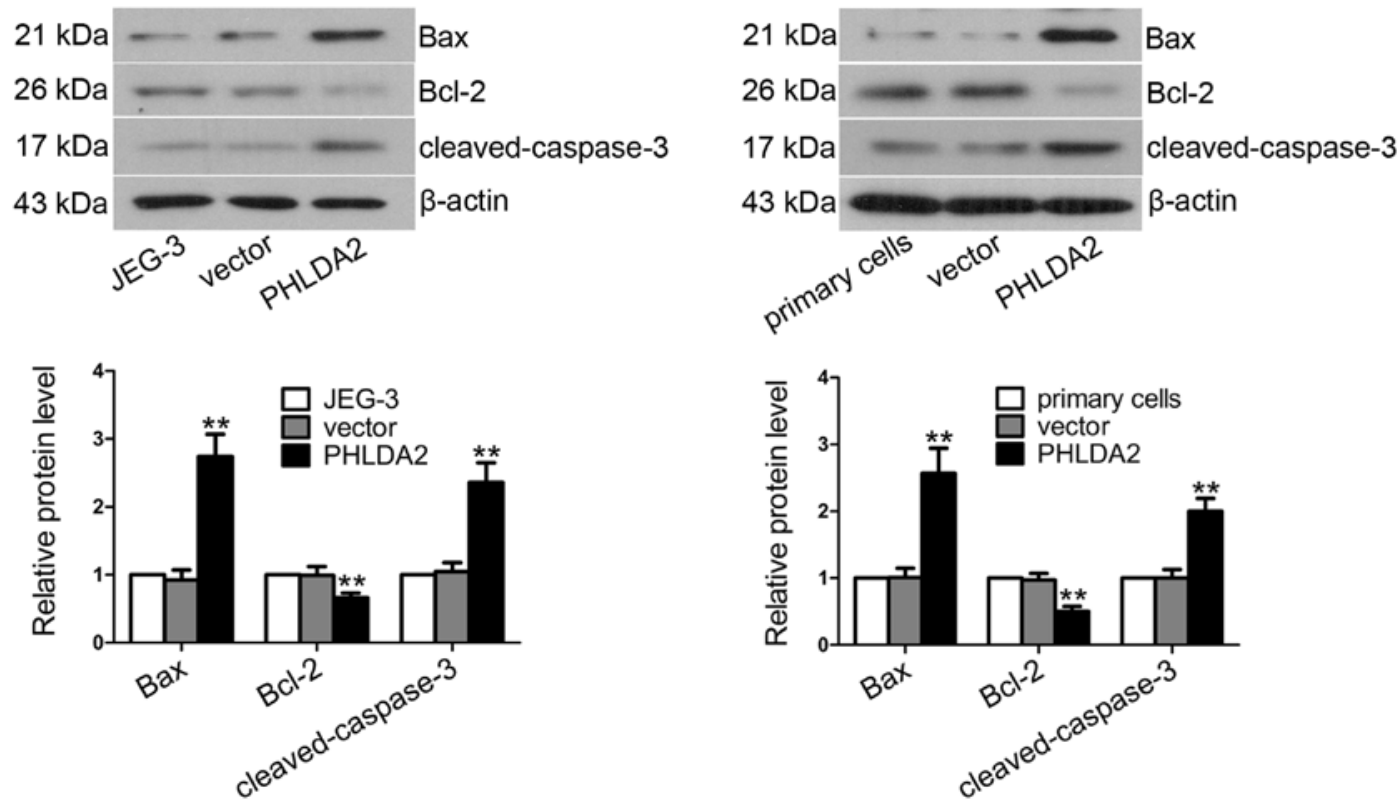

Figure 3. Pleckstrin homology-like domain, family A, member 2 (PHLDA2) overexpression promotes cell apoptosis. (A) After infection with lentiviruses for $24 \mathrm{~h}$, the apoptosis rate of JEG-3 cells and primary trophoblasts induced by PHLDA2 was measured using flow cytometry. (B) Apoptotic morphological changes in the JEG-3 and primary cells were detected by Hoechst staining and measured by fluorescence microscopy. Scale bar, $20 \mu \mathrm{m}$. (C) Proteins were extracted from the cells in each group and the concentrations were determined by BCA assay. Cleaved-caspase-3, Bax and Bcl-2 expression were analyzed by western blot analysis and normalized to $\beta$-actin expression. ${ }^{* *} \mathrm{P}<0.01$, compared with the control vector groups.

and 3.05-fold $(\mathrm{P}<0.01)$ higher than those in the JEG-3 cells infected with the lentivirus containing control vector. PHLDA2 mRNA and protein expression levels in the primary cells infected with PHLDA2-overexpressing lentivirus were increased to 3.38-fold $(\mathrm{P}<0.01)$ and 3.23-fold $(\mathrm{P}<0.01)$, respectively, when compared with levels in the primary cells infected with the vector-containing lentivirus.

PHLDA2 overexpression inhibits cell proliferation. To evaluate the effect of PHLDA2 overexpression in regulating the proliferation of JEG-3 cells and primary trophoblasts, a CCK- 8 cell proliferation assay was used at $24-\mathrm{h}$ post-infection. As shown in Fig. 2C, the proliferative capabilities at $48 \mathrm{~h}$ (JEG-3, $\mathrm{P}<0.05$; primary cells, $\mathrm{P}<0.01)$ and $72 \mathrm{~h}(\mathrm{JEG}-3, \mathrm{P}<0.05$; primary cells, $\mathrm{P}<0.05)$ were severely impaired in the JEG-3 and primary cells infected with the lentivurus overexpressing PHLDA2.

PHLDA2 overexpression promotes apoptosis. The ability of PHLDA2 to induce apoptosis was detected by Hoechst staining, flow cytometry and western blot analysis at $24 \mathrm{~h}$ post-infection. After the cells were stained with Annexin V-FITC/PI, a significant increase in the PHLDA2-induced apoptotic rate was observed by flow cytometric analysis in the JEG-3 cells infected with PHLDA2-overexpressing lentivirus $(25.37 \pm 4.11 \%$, 
A
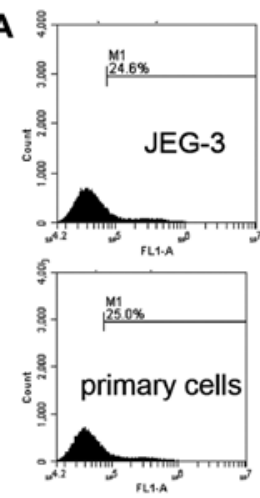

B

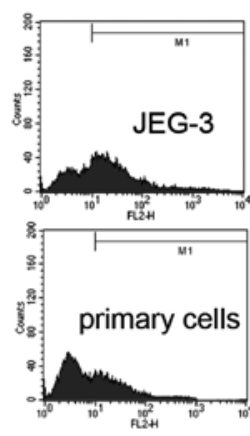

c
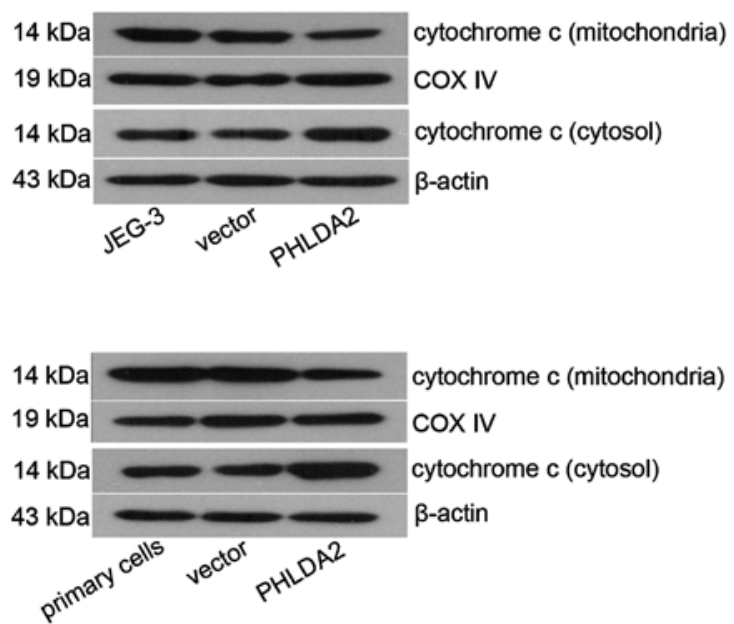
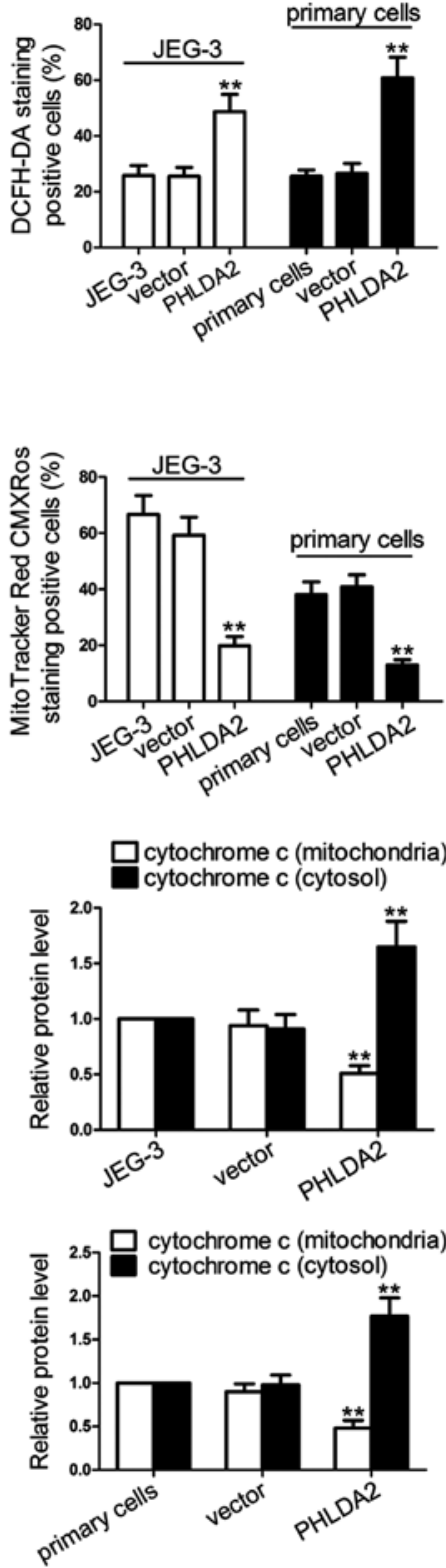

Figure 4. Pleckstrin homology-like domain, family A, member 2 (PHLDA2) overexpression induces mitochondrial injury. (A) Cells in each group were plated in a T25 culture flask, stained with DCFH-DA and incubated at $37^{\circ} \mathrm{C}$ for $20 \mathrm{~min}$. The cells were harvested and detected by flow cytometry. (B) JEG-3 cells and primary trophoblasts were stained with MitoTracker Red CMXRos and subjected to flow cytometric analysis. Mitochondrial membrane potential was then measured. (C) Mitochondrial and cytosolic proteins were obtained. Subsequently, cytochrome $c$ levels in the cytosol and mitochondria were detected using western blot analysis. COX IV was used as a loading control for mitochondrial proteins and $\beta$-actin for cytosolic proteins. ${ }^{* *} \mathrm{P}<0.01$, compared with the control vector groups.

$\mathrm{P}<0.01$ ) (Fig. 3A) and also the primary cells infected with PHLDA2-overexpressing lentivirus $(36.14 \pm 3.48 \%, \mathrm{P}<0.01)$ compared with those in the control vector group (JEG-3, $3.50 \pm 1.15 \%$; primary cells, $12.78 \pm 2.59 \%$ ), respectively. The condensed and fragmented nuclei were regarded as apoptotic cells. As observed from the photomicrographs, PHLDA2 overexpression markedly induced chromatin condensation (Fig. 3B). As shown in Fig. 3C, during the apoptotic process, PHLDA2 overexpression in JEG-3 cells and also in primary cells sharply increased cleaved-caspase-3 (JEG-3, 2.25-fold, $\mathrm{P}<0.01$; primary cells, 2.00-fold, $\mathrm{P}<0.01$ ) and pro-apoptotic protein Bax expression (JEG-3, 2.98-fold, $\mathrm{P}<0.01$; primary cells, 2.54 -fold, $\mathrm{P}<0.01$ ), respectively, compared with the vector-infected cells. Anti-apoptotic protein Bcl-2 levels were decreased 0.33-fold (JEG-3, P<0.01) and 0.48-fold (primary cells, $\mathrm{P}<0.01$ ).

PHLDA2 overexpression induces ROS production and also mitochondrial injury. To examine the impact of PHLDA2 overexpression on ROS accumulation, mitochondrial injury and cytochrome $c$ release, we measured mitochondrial membrane potential and ROS content using flow cytometric analysis at $24 \mathrm{~h}$ post-infection. Mitochondrial ROS was measured using DCFH-DA staining. The JEG-3 cells infected with PHLDA2-overexpressing lentivurus (Fig. 4A) $(\mathrm{P}<0.01)$ and the primary cells infected with PHLDA2-overexpressing 
A
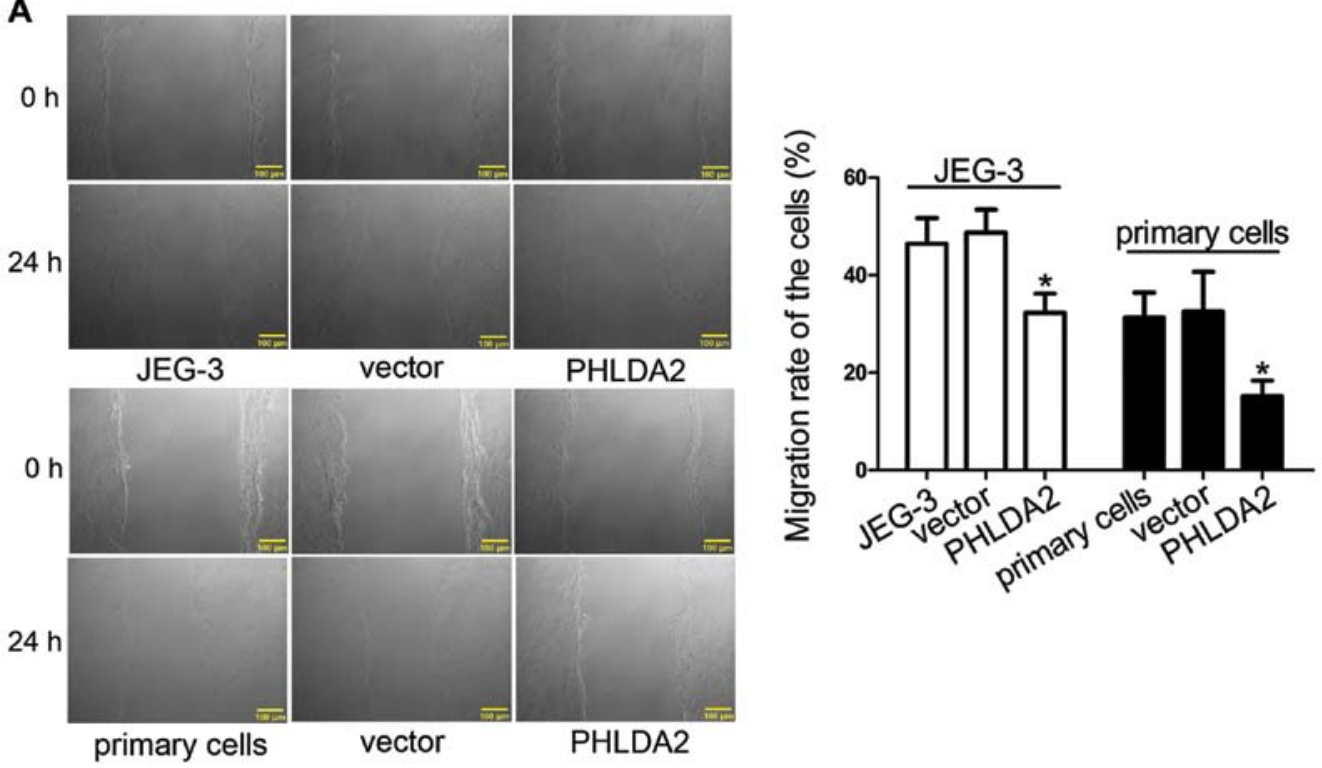

B
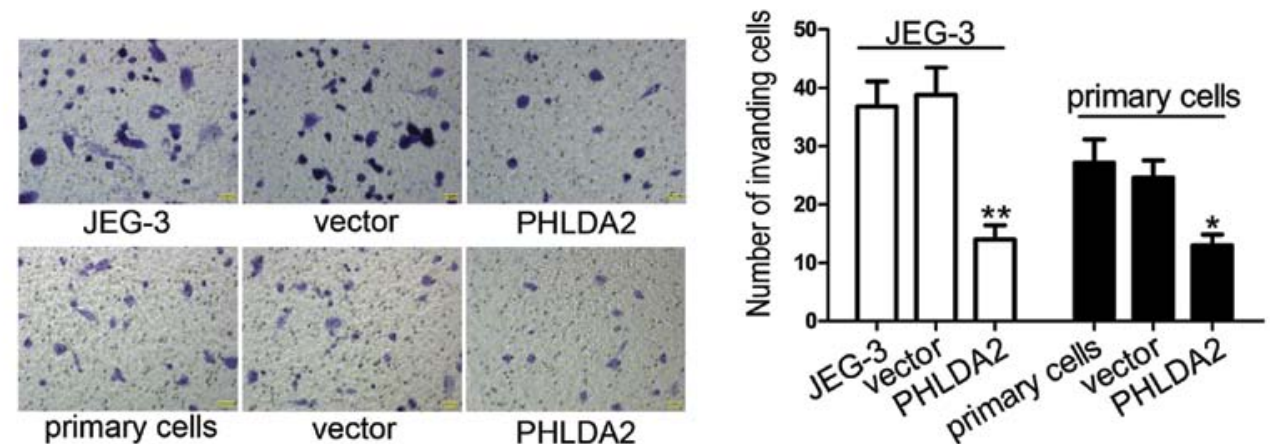

Figure 5. Pleckstrin homology-like domain, family A, member 2 (PHLDA2) overexpression suppresses cell migration and invasion. (A) The migration capability of JEG-3 cells and primary trophoblasts was evaluated by wound healing assay. Images were taken immediately and also $24 \mathrm{~h}$ after creating the scratch. The experiments were repeated three times. Scale bar, $100 \mu \mathrm{m}$. (B) Transwell assay. Representative images are shown. Invading cells were counted under a microscope. Scale bar, $50 \mu \mathrm{m} .{ }^{*} \mathrm{P}<0.05$ and ${ }^{* *} \mathrm{P}<0.01$, compared with the control vector groups.

lentivirus $(\mathrm{P}<0.01)$ demonstrated significantly increased ROS levels as compared with the groups infected with the vector-containing lentivirus. PHLDA 2 overexpression induced significant loss of mitochondiral membrane potential (JEG-3, $\mathrm{P}<0.01$; primary cells, $\mathrm{P}<0.01$ ) (Fig. 4B). Additionally, western blot analysis detected that cytochrome $c$ in the cytosol (JEG-3, 1.81-fold, $\mathrm{P}<0.01$; primary cells, 1.81-fold, $\mathrm{P}<0.01$ ) (Fig. 4C) was upregulated, whereas cytochrome $c$ in the mitochondria was downregulated (JEG-3, 0.46-fold, $\mathrm{P}<0.01$; primary cells, 0.47-fold, $\mathrm{P}<0.01)$.

PHLDA2 overexpression inhibits cell migration and invasion. To evaluate the role of PHLDA2 in the regulation of trophoblast migration, we carried out a wound healing assay at 24 h postinfection. The wound healing assay revealed that the migration rates of the cells in the JEG-3 group infected with PHLDA2overexpressing lentivirus $(32.30 \pm 3.93 \%, \mathrm{P}<0.05)$ (Fig. 5A) and the primary cells infected with PHLDA2-overexpressing lentivirus $(15.19 \pm 3.16 \%, \mathrm{P}<0.05)$ were significantly decreased compared with those treated with the control vector-containing lentivirus (JEG-3, 48.72 $\pm 4.73 \%$; primary cells, 32.59 $\pm 8.07 \%$ ). Subsequently, we assessed the effect of PHLDA2 on cell invasion with a Transwell assay. The results indicated that the number of invading cells in the JEG-3 group treated with lentivirus overexpressing PHLDA2 (14.00 2.45 cells/well, $\mathrm{P}<0.01$ ) (Fig. 5B) and the primary cells infected with PHLDA2overexpressing lentivirus $(13.00 \pm 1.87$ cells/well, $\mathrm{P}<0.05)$ were significantly lower than those in groups infected with the control

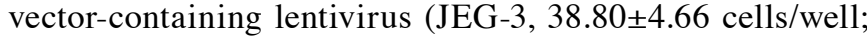
primary cells, $24.60 \pm 2.97$ cells/well).

\section{Discussion}

PHLDA2 is a maternally expressed and paternally imprinted gene (19) and is associated with fetal growth restriction (13). Our present study is the first, to the best of our knowledge, to demonstrate the impact of PHLDA2 on trophoblast function. We obtained primary trophoblasts, and CK18 (20), vimentin and hPL (21) were used as markers to characterize and identify trophoblasts. We detected high expression levels of CK18, vimentin and hPL in primary trophoblasts with immunofluorescence staining. Subsequently, primary trophoblasts and JEG-3 cells were infected with lentiviruses and PHLDA2 overexpression cell lines were established. We noted that PHLDA2 inhibited cell proliferation, migration and invasion, and induced cell apoptosis.

Bcl-2 family members act as regulators of cell apoptosis (22) and have been divided into three groups: 
antiapoptotic proteins (Bcl-2, Bcl-xl and $\mathrm{Mcl}-1)$, proapoptotic proteins (Bax and Bak) and BH3-only proteins (Bad, Bik and Bid) (23). Once the cells receive apoptosis signals, proapoptotic proteins Bax and Bak are activated (oligomerization of Bax and Bak), inserted into the outer mitochondrial membrane and then trigger the release of cytochrome $c$ into the cytosol. Subsequently, cytochrome $c$ activates caspases and induces cell apoptosis $(24,25)$. The role of antiapoptotic members is to inhibit their proapoptotic partners (26). A previous study has reported that PHLDA2 inhibits tumor growth and induces tumor cell apoptosis in vivo. Knockdown of PHLDA2 promotes human osteosarcoma SaOS-2 cell proliferation and decreases the apoptotic rate in vitro and in vivo (27). However, the roles of PHLDA2 in the proliferation and apoptosis of trophoblasts have not yet been reported. In the present study, JEG-3 cells and primary trophoblasts were infected with lentiviruses. We then evaluated the impact of PHLDA2 overexpression on cell proliferation capability and apoptosis. The results showed that PHLDA2 mRNA and protein expression levels were remarkably upregulated in both sets of cells infected with the PHLDA2-overexpressing lentivirus. In accordance with previous research (27), the overexpression of PHLDA2 significantly suppressed cell proliferation and induced apoptosis, and the activation of caspase- 3 and pro-apoptotic protein Bax, as well as the inhibition of antiapoptotic protein Bcl-2.

ROS, such as superoxide anions, hydroxyl radicals and hydrogen peroxide, are the most common metabolic products of organism (28). Low levels of ROS serve as 'redox messengers' in the regulation of multiple signaling pathways and cellular processes, whereas excess ROS lead to the inhibition of protein functions and the promotion of cell apoptosis (29). When ROS reach a threshold level, they cause a decrease in mitochondrial membrane potential, and also cause the release of cytochrome $c$ from the mitochondria into the cytosol and the release of caspase-activating proteins $(29,30)$. PHLDA2 has been shown to induce apoptosis of osteosarcoma tumorinitiating cells by activating caspase- 3 , releasing cytochrome $c$ and decreasing the mitochondrial membrane potential (31). The results of the present study demonstrated that PHLDA2 significantly promoted the release of cytochrome $c$, and induced loss of mitochondrial membrane potential and ROS accumulation in trophoblasts. Our study suggests that PHLDA2 overexpression induces trophoblast apoptosis via the mitochondrial pathway and the accumulation of ROS.

The invasion of trophoblasts into the maternal endometrium plays an important role in successful implantation and placentation during pregnancy (32-36). Various researchers have demonstrated that shallow trophoblast invasion and inadequate uterine remodeling of spiral arteries are ubiquitous in the placenta of patients with PE in previous studies $(37,38)$. Additionally, the expression of PHLDA2 is elevated in the placenta of infants who are affected by IUGR (10). PHLDA2 is a regulator of embryonic development. It has previously been suggested that PHLDA2 overexpression results in placental abnormalities, including IUGR and PE, whereas low expression of PHLDA2 causes hypertrophic placenta or placentomegaly (39). Based on these statements, we hypothesized that PHLDA2 was associated with trophoblast invasion. Therefore, in the present study, trophoblasts were infected with a lentivirus overexpressing PHLDA2 in order to examine the functions of PHLDA2. As expected, our results demonstrated that PHLDA2 overexpression inhibited trophoblast migration and invasion.

Our results indicate that PHLDA2 overexpression inhibited trophoblast proliferation and induced cell apoptosis through the involvement of the mitochondrial pathway. Moreover, we suggest that PHLDA2 inhibits trophoblast migration and invasion. These results suggest that PHLDA2 overexpression causes inadequate trophoblast invasion. Further studies are warranted to validate our results. Our study provides evidence for investigating the possible role of PHLDA2 in the occurence and progression of pregnancy-associated complications.

\section{References}

1. Cha J, Sun X and Dey SK: Mechanisms of implantation: strategies for successful pregnancy. Nat Med 18: 1754-1767, 2012.

2. Garnica AD and Chan WY: The role of the placenta in fetal nutrition and growth. J Am Coll Nutr 15: 206-222, 1996.

3. Nagashima T, Li Q, Clementi C, Lydon JP, DeMayo FJ and Matzuk MM: BMPR2 is required for postimplantation uterine function and pregnancy maintenance. J Clin Invest 123: 2539-2550, 2013.

4. Romero R, Kusanovic JP, Chaiworapongsa T and Hassan SS: Placental bed disorders in preterm labor, preterm PROM, spontaneous abortion and abruptio placentae. Best Pract Res Clin Obstet Gynaecol 25: 313-327, 2011.

5. Whitley GS, Dash PR, Ayling LJ, Prefumo F, Thilaganathan B and Cartwright JE: Increased apoptosis in first trimester extravillous trophoblasts from pregnancies at higher risk of developing preeclampsia. Am J Pathol 170: 1903-1909, 2007.

6. Valenzuela FJ, Vera J, Venegas C, Pino F and Lagunas C: Circadian system and melatonin hormone: risk factors for complications during pregnancy. Obstet Gynecol Int 2015: 825802, 2015.

7. Khong Y and Brosens I: Defective deep placentation. Best Pract Res Clin Obstet Gynaecol 25: 301-311, 2011.

8. Huang Y, Dong F, Du Q, Zhang H, Luo X, Song X, Zhao X, Zhang $\mathrm{W}$ and Tong D: Swainsonine induces apoptosis through mitochondrial pathway and caspase activation in goat trophoblasts. Int J Biol Sci 10: 789-797, 2014.

9. Qian N, Frank D, O'Keefe D, Dao D, Zhao L, Yuan L, Wang Q, Keating M, Walsh C and Tycko B: The IPL gene on chromosome $11 \mathrm{p} 15.5$ is imprinted in humans and mice and is similar to TDAG51, implicated in Fas expression and apoptosis. Hum Mol Genet 6: 2021-2029, 1997.

10. McMinn J, Wei M, Schupf N, Cusmai J, Johnson EB, Smith AC, Weksberg R, Thaker HM and Tycko B: Unbalanced placental expression of imprinted genes in human intrauterine growth restriction. Placenta 27: 540-549, 2006.

11. Jensen AB, Tunster SJ and John RM: The significance of elevated placental PHLDA2 in human growth restricted pregnancies. Placenta 35: 528-532, 2014.

12. Apostolidou S, Abu-Amero S, O'Donoghue K, Frost J, Olafsdottir O, Chavele KM, Whittaker JC, Loughna P, Stanier P and Moore GE: Elevated placental expression of the imprinted PHLDA2 gene is associated with low birth weight. J Mol Med Berl 85: 379-387, 2007.

13. Tunster SJ, Van De Pette M and John RM: Isolating the role of elevated Phlda2 in asymmetric late fetal growth restriction in mice. Dis Model Mech 7: 1185-1191, 2014.

14. Kumar N, Leverence J, Bick D and Sampath V: Ontogeny of growth-regulating genes in the placenta. Placenta 33: 94-99, 2012.

15. Diplas AI, Lambertini L, Lee MJ, Sperling R, Lee YL, Wetmur J and Chen J: Differential expression of imprinted genes in normal and IUGR human placentas. Epigenetics 4: 235-240, 2009.

16. Frank D, Fortino W, Clark L, Musalo R, Wang W, Saxena A, Li CM, Reik W, Ludwig T and Tycko B: Placental overgrowth in mice lacking the imprinted gene Ipl. Proc Natl Acad Sci USA 99: 7490-7495, 2002.

17. Driver AM, Huang W, Kropp J, Peñagaricano F and Khatib H: Knockdown of $C D K N 1 C$ (p57(kip2)) and PHLDA2 results in developmental changes in bovine pre-implantation embryos. PLoS One 8: e69490,2013.

18. Livak KJ and Schmittgen TD: Analysis of relative gene expression data using real-time quantitative PCR and the 2(-Delta Delta C(T)) method. Methods 25: 402-408, 2001. 
19. Demetriou C, Abu-Amero S, Thomas AC, Ishida M, Aggarwal R Al-Olabi L, Leon LJ, Stafford JL, Syngelaki A, Peebles D, et al: Paternally expressed, imprinted insulin-like growth factor- 2 in chorionic villi correlates significantly with birth weight. PLoS One 9: e85454, 2014.

20. Zhang L, Zhang W, Shao C, Zhang J, Men K, Shao Z, Yan Y and Xu D: Establishment and characterization of a spontaneously immortalized trophoblast cell line (HPT-8) and its hepatitis B virus-expressing clone. Hum Reprod 26: 2146-2156, 2011.

21. Aboagye-Mathiesen G, Laugesen J,Zdravkovic M and Ebbesen P: Isolation and characterization of human placental trophoblast subpopulations from first-trimester chorionic villi. Clin Diagn Lab Immunol 3: 14-22, 1996.

22. Liu Z, Lu H, Jiang Z, Pastuszyn A and Hu CA: Apolipoprotein 16, a novel proapoptotic $\mathrm{Bcl}-2$ homology 3 -only protein, induces mitochondria-mediated apoptosis in cancer cells. Mol Cancer Res 3: 21-31, 2005.

23. Brinkmann $\mathrm{K}$ and Kashkar H: Targeting the mitochondrial apoptotic pathway: a preferred approach in hematologic malignancies? Cell Death Dis 5: e1098, 2014.

24. Green DR and Kroemer G: The pathophysiology of mitochondrial cell death. Science 305: 626-629, 2004.

25. Adams JM and Cory S: The Bcl-2 apoptotic switch in cancer development and therapy. Oncogene 26: 1324-1337, 2007.

26. Hardwick JM and Soane L: Multiple functions of BCL-2 family proteins. Cold Spring Harb Perspect Biol 5: 5, 2013.

27. Dai H, Huang Y, Li Y, Meng G, Wang Y and Guo QN: TSSC3 overexpression associates with growth inhibition, apoptosis induction and enhances chemotherapeutic effects in human osteosarcoma. Carcinogenesis 33: 30-40, 2012.

28. Xu Q, Zhang B, Li XM and Gao X: Traditional Chinese medicine formula Qing Huo Yi Hao as superoxide anion scavenger in high glucose-treated endothelial cells. Acta Pharmacol Sin 33 : 496-502, 2012

29. Circu ML and Aw TY: Reactive oxygen species, cellular redox systems, and apoptosis. Free Radic Biol Med 48: 749-762, 2010.

30. Zorov DB, Juhaszova M and Sollott SJ: Mitochondrial ROSinduced ROS release: an update and review. Biochim Biophys Acta 1757: 509-517, 2006.
31. Huang Y, Dai H and Guo QN: TSSC3 overexpression reduces stemness and induces apoptosis of osteosarcoma tumor-initiating cells. Apoptosis 17: 749-761, 2012.

32. Pijnenborg R, Robertson WB, Brosens I and Dixon G: Review article: trophoblast invasion and the establishment of haemochorial placentation in man and laboratory animals. Placenta 2: 71-91, 1981

33. Aplin JD: Implantation, trophoblast differentiation and haemochorial placentation: mechanistic evidence in vivo and in vitro. J Cell Sci 99: 681-692, 1991.

34. Bischof P, Meisser A and Campana A: Biochemistry and molecular biology of trophoblast invasion. Ann N Y Acad Sci 943: 157-162, 2001.

35. Tapia-Pizarro A, Argandoña F, Palomino WA and Devoto L: Human chorionic gonadotropin (hCG) modulation of TIMP1 secretion by human endometrial stromal cells facilitates extravillous trophoblast invasion in vitro. Hum Reprod 28: 2215-2227, 2013

36. Yang Q, Chen SP, Zhang XP, Wang H, Zhu C and Lin HY: Smurf2 participates in human trophoblast cell invasion by inhibiting TGF-beta type I receptor. J Histochem Cytochem 57: 605-612, 2009.

37. Lim KH, Zhou Y, Janatpour M, McMaster M, Bass K, Chun SH and Fisher SJ: Human cytotrophoblast differentiation/invasion is abnormal in pre-eclampsia. Am J Pathol 151: 1809-1818, 1997.

38. George EM and Granger JP: Recent insights into the pathophysiology of preeclampsia. Expert Rev Obstet Gynecol 5: 557-566, 2010.

39. Salas M, John R, Saxena A, Barton S, Frank D, Fitzpatrick G, Higgins MJ and Tycko B: Placental growth retardation due to loss of imprinting of Phlda2. Mech Dev 121: 1199-1210, 2004. 